\title{
The migration of neighboring and antagonist teeth three months after implant placement in healed single tooth-missing sites
}

\author{
Guo, Danni ; Pan, Shaoxia ; Mühlemann, Sven ; Zhou, Yongsheng ; Jung, Ronald Ernst
}

\begin{abstract}
Objectives: To quantify the neighboring and antagonist teeth migration of a single posterior toothmissing site within 3 months using digital scanning and measuring techniques. Materials and methods: Intraoral scans (IOS) were made in 40 patients presenting a single posterior tooth-missing gap and receiving implant therapy. IOS were obtained at the day of and three months after implant surgery rendering a digital baseline model (BM) and a digital follow-up model (FM). Digital models were superimposed using the implant scan body as reference. Antagonist models were processed by the best fit alignment. Dimensional change between anatomical landmarks on neighboring teeth and that of featuring points on antagonistic teeth were measured using a threedimensional analysis software. The Mann-Whitney U test was applied to compare the tooth-moving distance between the mesial and distal neighboring teeth. The Kruskal-Wallis one-way ANOVA was used to test the difference in dimensional change in tooth-missing site among age subgroups. Results: The mean dimensional change in the tooth-missing site was $-37.62 \pm 106.36 \mu \mathrm{m}$ (median: -28.33 $\mu \mathrm{m}, \mathrm{Q} 25-72.65 / \mathrm{Q} 75$ 38.97) mesial-distally and $-67.91 \pm 42.37 \mu \mathrm{m}$ (median: $-61.50 \mu \mathrm{m}, \mathrm{Q} 25-88.25 / \mathrm{Q} 75-36.75$ ) occlusal-gingivally. Eighteen out of 40 mesial neighboring teeth and 24 out of 40 distal neighboring teeth showed migration towards the implants. When patients were grouped according to age, the mesial-distal reduction in the tooth-missing site was significantly larger in patients younger than 30 years compared with those older than 50 years $(\mathrm{p}<.05)$. Conclusions: The dimensions of posterior tooth-missing sites decreased over an observation period of 3 months. Keywords: dental implants; digital; three-dimensional; tooth migration; tooth movement.
\end{abstract}

DOI: https://doi.org/10.1111/clr.13694

Posted at the Zurich Open Repository and Archive, University of Zurich

ZORA URL: https://doi.org/10.5167/uzh-194796

Journal Article

Accepted Version

Originally published at:

Guo, Danni; Pan, Shaoxia; Mühlemann, Sven; Zhou, Yongsheng; Jung, Ronald Ernst (2021). The migration of neighboring and antagonist teeth three months after implant placement in healed single tooth-missing sites. Clinical Oral Implants Research, 32(2):233-241.

DOI: https://doi.org/10.1111/clr.13694 
DR. SHAO-XIA PAN (Orcid ID : 0000-0002-3808-9499)

DR. SVEN MÜHLEMANN (Orcid ID : 0000-0003-1253-1813)

PROF. YONGSHENG ZHOU (Orcid ID : 0000-0002-4332-0878)

DR. RONALD ERNST JUNG (Orcid ID : 0000-0003-2055-1320)

Article type : Original Research

\section{The migration of neighboring and antagonist teeth three months after implant}

\section{placement in healed single tooth-missing sites}

Running Title: Neighboring teeth migration after implant placement

Danni Guo ${ }^{1}$, Shaoxia Pan ${ }^{*}$, Sven Mühlemann², Yongsheng Zhou' ${ }^{1}$, Ronald Jung²

1.Department of Prosthodontics, Peking University School and Hospital of Stomatology, National Clinical Research Center for Oral Diseases, National Engineering Laboratory for Digital and Material Technology of Stomatology, Beijing Key Laboratory of Digital Stomatology, Beijing, P.R. China.

2. Clinic of Reconstructive Dentistry, Center of Dental Medicine, University of Zurich, Zurich, Switzerland

*Corresponding author: Dr. Shaoxia Pan, Email: panshaoxia@vip.163.com

Mailing address: Department of Prosthodontics, Peking University School and Hospital of Stomatology, No. 22 Zhongguancun South Avenue, Haidian District, Beijing 100081, P.R. China. Tel: (86)13011110951, Fax: (8610)62173402

This article has been accepted for publication and undergone full peer review but has not been through the copyediting, typesetting, pagination and proofreading process, which may lead to differences between this version and the Version of Record. Please cite this article as doi: $\underline{10.1111 / C L R .13694}$

This article is protected by copyright. All rights reserved 


\section{Authors' contributions}

S.P., S.M., R.J., Y.Z. conceived the ideas; S.P. and D.G. collected the data; S.P., D.G., Y.Z. analyzed the data; and S.P., S.M., R.J., Y.Z. led the writing. 


\section{Abstract}

Objectives: To quantify the neighboring and antagonist teeth migration of a single posterior tooth-missing site within three months using digital scanning and measuring techniques.

Material and Methods: Intraoral scans (IOS) were made in 40 patients presenting a single posterior tooth-missing gap and receiving implant therapy. IOS were obtained at the day of and three months after implant surgery rendering a digital baseline model (BM) and a digital follow-up model (FM). Digital models were superimposed using the implant scan body as reference. Antagonist models were processed by the best fit alignment. Dimensional change between anatomical landmarks on neighboring teeth, and that of featuring points on antagonistic teeth were measured using a three-dimensional analysis software. The Mann-Whitney U test was applied to compare the tooth moving distance between the mesial and distal neighboring teeth. The Kruskal-Wallis one-way ANOVA was used to test the difference in dimensional change of tooth-missing site among age subgroups.

Results: The mean dimensional change of the tooth-missing site was $-37.62 \pm 106.36 \mu \mathrm{m}$ (median: $-28.33 \mu \mathrm{m}, \mathrm{Q}_{25}-72.65 / \mathrm{Q}_{75} 38.97$ ) mesial-distally and $-67.91 \pm 42.37 \mu \mathrm{m}$ (median: $-61.50 \mu \mathrm{m}, \mathrm{Q}_{25}$ $\left.-88.25 / \mathrm{Q}_{75}-36.75\right)$ occlusal-gingivally. Eighteen out of 40 mesial neighboring teeth and 24 out of 40 distal neighboring teeth showed migration towards the implants. When patients were grouped according to age, the mesial-distal reduction of the tooth-missing site was significantly larger in patients younger than 30 years compared to those older than 50 years $(P<0.05)$.

Conclusions: The dimensions of posterior tooth-missing sites decreased over an observation period of three months.

Key words: tooth migration, tooth movement, dental implants, three-dimensional, digital

This article is protected by copyright. All rights reserved 


\section{Introduction}

The reports about migration of neighboring and antagonist teeth after natural tooth extraction remain inconsistent. A variety of clinical studies revealed that the integrity and stability of natural dentition could be affected for a long time after tooth extraction if the space was left without restoration (Christou \& Kiliaridis, 2007; Craddock, Youngson, Manogue, \& Blance, 2007; Garcia-Herraiz, Leiva-Garcia, Canigral-Ortiz, Silvestre, \& Garcia-Anton, 2012; Garcia-Herraiz, Silvestre, Leiva-Garcia, Crespo-Abril, \& Garcia-Anton, 2017).

Drifting and tipping of the neighboring teeth (Weinstei.S, 1967) and overeruption of the antagonist teeth (Lyka, Carlsson, Wedel, \& Kiliaridis, 2001) are typical phenomena observed 5 to 12 years after tooth loss (Christou \& Kiliaridis, 2007; Lindskog-Stokland, Hansen, Tomasi, Hakeberg, \& Wennstrom, 2012). Nevertheless, other studies showed that the incidence of adjacent teeth moving toward edentulous area was not always the same (Kiliaridis, Lyka, Friede, Carlsson, \& Ahlqwist, 2000; Love \& Adams, 1971), and the probability and distance of teeth movement could be small. The mean reduction of the mesial-distal tooth-missing gap did not exceed $1 \mathrm{~mm}$ six years after tooth extraction (Gragg, Shugars, Bader, Elter, \& White, 2001; Shugars, Bader, Phillips, White, \& Brantley, 2000). With the tooth missing time prolonged, the risk of dimensional edentulous gap changes decreased (Garcia-Herraiz et al., 2017; Gragg et al., 2001).

Most of the studies investigating dimensional change of tooth-missing gap after tooth extraction generally had a 5 to 20 year observation time (Christou \& Kiliaridis, 2007; Gragg et al., 2001; Kiliaridis et al., 2000; Love \& Adams, 1971; Shugars et al., 2000). Only one study compared the change of tooth-missing gap between one month and three months after tooth extraction by analyzing stone models with confocal laser scanning microscopy technique (Garcia-Herraiz et al., 2017). They reported $0.35 \mathrm{~mm}$ and $0.67 \mathrm{~mm}$ mean gap reduction one and three months following tooth extraction.

Different outcomes may come from different timing and measurement techniques. Methods of measurement used in prior studies included: clinical examination (Kiliaridis et al., 2000; Love \& Adams, 1971), questionnaire(Kiliaridis et al., 2000), radiographic evaluation (Gragg et al., 2001; Lindskog-Stokland et al., 2012; Shugars et al., 2000), stone model measurement 
(Garcia-Herraiz et al., 2017). Some of these methods were qualitative techniques (Kiliaridis et al., 2000; Love \& Adams, 1971), while others were quantitative techniques (Garcia-Herraiz et al., 2017; Gragg et al., 2001; Lindskog-Stokland et al., 2012; Shugars et al., 2000). These quantitative techniques have some limitations such as lacking fixed reference markers, using two-dimensional (2D) radiographies, involving too many manual interventions in data acquisition, or failing to standardize the reference points. All of these factors may have an influence on the results.

Novel digital technology has been adopted to improve dimensional measurement accuracy. Recent studies showed that the fully digital workflow in the restoration of posterior single implant crowns was highly effective and efficient (Joda, Ferrari, \& Bragger, 2017; Muhlemann, Kraus, Hammerle, \& Thoma, 2018). Intraoral scanners with an accuracy of up to $12.7 \mu \mathrm{m}$ (Moreira, Rodrigues, Pinho, Fonseca, \& Vilaca, 2015) can generate digital model which can be easily stored, transmitted, and utilized repeatedly without information loss over time (Shah, Sundaram, Bartlett, \& Sherriff, 2004). Compared with 2D radiographic measurement, linear measurement of three-dimensional (3D) digital model and quantitative analysis using inspection software can reduce patients' X-ray exposure and the error caused by image deformation.

The latest research showed that optical impression could be taken immediately after implant placement, and posterior single implant crown could be successfully delivered three months after the surgery. (Guo et al., 2019; Pan et al., 2019). However, it's still unclear if there are dimensional changes of the tooth-missing site during the three months following implant placement.

Therefore, the aim of the present clinical study was to analyze position changes of neighboring and antagonist teeth of single posterior tooth-missing site, between the day of implant placement and three months later using digital scanning and measuring techniques. The null hypothesis was that there were no dimensional changes of single posterior tooth-missing sites between implant placement and three months later.

\section{Material and methods}

1. Participants

This study was conducted in the Department of Prosthodontics, Peking University School and 


\section{Hospital of Stomatology.}

The study was reviewed and approved by the Institutional Review Board of Peking University School and Hospital of Stomatology (Ethical approval No: PKUSSIRB-201732002). The study had been registered in Chinese Clinical Trial Registry (ChiCTR) (ChiCTR No: INR-17014092). The Consolidated Standards of Reporting Trials (CONSORT) guidelines were used as the framework for this study.

This study was undertaken with the understanding and written consent of each subject and according to the World Medical Association Declaration of Helsinki (version 2013).

The inclusion criteria were:

- Age $\geq 18$ years old

- Missing single posterior premolar or first molar for at least three months

- Mesial and distal teeth / fixed restorations present and intact

- Sufficient bone height and width at implant site (vertical bone height $\geq 10 \mathrm{~mm}$, buccal-lingual bone width $\geq 6 \mathrm{~mm}$ )

- Sufficient prosthetic space (Vertical height $\geq 5 \mathrm{~mm}$, mesial-distal distance $\geq 6 \mathrm{~mm}$ )

- Willing to receive implant treatment

The exclusion criteria were:

- Local or systemic contraindication for implant therapy (i.e. uncontrolled diabetes, hemophilia, metabolic bone disorder, history of renal failure, radiation treatment to the head or neck region, current chemotherapy, and pregnancy etc.)

- $\quad$ Smoking $\geq 10$ cigarettes per day

- In need for major guided bone regeneration (GBR) / submucosal implant healing

2. Clinical examination and implant placement

The surgical procedure of implant placement was described in a previous article reporting on the same patient cohort (Pan et al., 2019). In brief, at the first visit a clinical and radiographic examination (CBCT, VGi evo, NewTom) was performed. An intraoral scan (IOS) including both jaws and a bite registration (3Shape Trios ${ }^{\circledR}$ Standard-P11, 3Shape A/S) were made. At the second appointment, the implant (Straumann Bone Level or Tissue Level, Institut Straumann AG) was 
placed according to the manufacturers' instructions.

3. Digital model acquisition

Immediately after implant placement, an implant-specific scan body (Institut Straumann AG) was connected onto the implant, and the intraoral scan acquired during the first visit was updated with the scan body (Figure 1a). Three months following implant placement, a second intraoral scan was taken including both jaws, the implant site with the scan body in the same alignment as during the first scan, and the bite registration. (Figure $1 \mathrm{~b}$ ).

Digital models were generated from the optical impression datasets and saved in Stereolithography (STL) format by means of a Computer-aided design (CAD) software (3Shape Designer, 3Shape A/S). Digital models obtained at the day of surgery were considered as the baseline models (BM) and digital models generated three months following implant placement were considered as the follow-up models (FM). The two digital models were imported into a 3D analysis software (Geomagic Qualify 2012, 3D SYSTEMS) for the dimensional measurements.

4. Three-dimensional measurement of neighboring teeth migration

1) Determination of reference lines on the neighboring teeth

On the BM, a buccal plane was generated according to a buccal line connecting the most prominent point on the buccal surface of the mesial and distal neighboring teeth and paralleling to the central axis of the scan body, then a reference plane (RP) was determined parallel to the buccal plane and running through the central axis of the scan body (Fig. 2a). Two reference lines were obtained in the RP by drawing lines parallel to the central axis of the scan body and tangential to the mesial and distal outline of the neighboring teeth (Fig. 2b). The scan body of the FM was superimposed on the scan body of the BM using best fit alignment. The RP in the BM was replicated in the FM, and used to determine the mesial and distal reference lines in the FM.

2) Measurement of neighboring tooth movement in the RP

The distances between the central axis of the scan body and the parallel reference lines were measured mesially (D1) and distally (D2) in both the BM and the FM (Fig. 2c). The total mesial-distal dimensional change of the tooth-missing site $\left[\left(\mathrm{D} 1_{\mathrm{FM}}+\mathrm{D} 2_{\mathrm{FM}}\right)-\left(\mathrm{D} 1_{\mathrm{BM}}+\mathrm{D} 2_{\mathrm{BM}}\right)\right]$, the mesial neighboring tooth migration $\left(\mathrm{D} 1_{\mathrm{BM}}-\mathrm{D} 1_{\mathrm{FM}}\right)$, and the distal neighboring tooth migration 
$\left(\mathrm{D} 2_{\mathrm{BM}}-\mathrm{D} 2_{\mathrm{FM}}\right)$ were calculated.

5. Three-dimensional measurement of antagonist teeth migration

1) Determination of reference points on antagonist tooth

Five featuring points were selected on the occlusal surface of the antagonist tooth opposite to the tooth-missing site (Figure 3). In case of two antagonist teeth opposing the site, the one with the larger occlusal area exposed to the tooth-missing gap was chosen as the antagonist of interest.

2) Measurement of antagonist tooth migration

A $3 \mathrm{D}$ coordinate system was established on the BM of the opposing dentition by defining a horizontal plane with three landmark points: the incisal point of mesial interproximal contact of the central incisors and the two mesial buccal cusp tips of the first molar on each side. Then the $\mathrm{z}$-axis was set perpendicular to the horizontal plane. The BM and the FM of the opposing dentition were superimposed using best fit alignment of the neighboring teeth to the antagonist tooth. The standard of best fit registration was as follow: after the best fit alignment of the selected area with 100,000 sampling size the root mean square error (RMS) was less than $30 \mu \mathrm{m}$ (Peters et al., 1999).

The vector change in the z-axis direction of the five featuring points on the antagonist tooth between the BM and the FM were recorded, and the mean vector change of the five points was calculated to represent the migration distance of the antagonist tooth (Figure 3a, b, c). This is also the occlusal-gingival dimensional change of the tooth missing site. Average occlusal-gingival change of the tooth missing site in the cohort was then calculated.

6. Statistical analysis

A power analysis was performed using the data originated from a previous study assessing mesial-distal dimensional change of tooth-missing gap three months post extraction. The power analysis was based on an independent t-test. A sample size of 22 will have $90 \%$ power to detect a gap reduction of $613 \mu \mathrm{m}$, assuming a standard deviation of $698 \mu \mathrm{m}$ (Garcia-Herraiz et al., 2012). Considering the possibility of $20 \%$ loss of follow-up rate, the sample size of this study was set at 40.

All statistical analyses were conducted in SPSS software (IBM SPSS Statistics v22; IBM Corp). The results of Shapiro-Wilk test showed that some of the data were not normally 
distributed, therefore the nonparametric Mann-Whitney U test was used to compare tooth moving distance between the mesial and distal neighboring teeth groups, male and female, premolar and molar, and upper and lower jaws. The Kruskal-Wallis one-way ANOVA was used to test the difference in dimensional change of tooth-missing site among age subgroups. The level of significance was set at 0.05 .

\section{Results}

Forty participants with 21 females and 19 males were recruited in this study, and the mean age was 45.1 years. The tooth-missing position and numbers are shown in Table 1. The mean tooth-missing time was 14 months (range: 3-84 months).

Three months after implant surgery, the mean mesial-distal dimensional change of the tooth-missing site was $-37.62 \pm 106.36 \mu \mathrm{m}$ (median: $-28.33 \mu \mathrm{m}, \mathrm{Q}_{25}-72.65 / \mathrm{Q}_{75} 38.97$, the negative value represents a distance reduction). In 25 participants, the mesial-distal distance of the tooth-missing site decreased, while in the other 15 patients, the mesial-distal distance increased.

For the mesial neighboring teeth, 18 migrated toward while 22 away from the implant. For the distal neighboring teeth, 24 migrated towards and 16 away from the implant. Table 2 shows the median, the $\mathrm{Q}_{25}$, and the $\mathrm{Q}_{75}$ of the mesial and distal moving distance of the mesial and distal neighboring teeth. The distal moving distance of the mesial neighboring teeth was significantly larger than that of the distal neighboring teeth $(P=0.003)$.

The antagonist teeth extrude towards the tooth-missing sites in all forty patients, and the average occlusal-gingival change of the tooth missing site was $-67.91 \pm 42.37 \mu \mathrm{m}$ (median: $-61.50 \mu \mathrm{m}, \mathrm{Q}_{25}-88.25 / \mathrm{Q}_{75}-36.75$, the negative value represents a distance reduction).

Participants were divided into three age subgroups (under 30 years, 30 to 50 years, and above 50 years), the mesial-distal reduction of the tooth-missing site was significantly larger in patients younger than 30 years compared to those older than 50 years (Table 3, Figure 4). No significant difference was found in occlusal gingival dimensional change of the tooth-missing site among the three subgroups (Table 3, Figure 4).

There was no significant difference in the mesial-distal and occlusal-gingival dimensional change of tooth-missing site between sexes (male or female), tooth positions (molar or premolar), 
and jaws (maxilla or mandible) during the three months after implant placement (Table 4).

\section{Discussion}

The null hypothesis stating that there were no dimensional changes of single posterior tooth-missing sites between implant placement and three months later was rejected. The mean mesial-distal dimensional change of posterior single tooth-missing sites was $-37.62 \pm 106.36 \mu \mathrm{m}$, with 25 out of 40 participants demonstrating mesial-distal distance reduction while 15 participants showing mesial-distal distance increase. The occlusal-gingival distance reduction of the posterior single tooth-missing site was observed in all 40 patients with a mean change of $-67.91 \pm 42.37 \mu \mathrm{m}$.

This study investigated the short-term dimensional changes in single posterior tooth-missing site. A conventional loading protocol of taking impression 3 months after implant placement has been adopted in this study. According to the systematic review (Gallucci, Hamilton, Zhou, Buser, $\&$ Chen, 2018) reported on the $6^{\text {th }}$ ITI concensus conference, when implants were placed in healed sites, the loading protocols (early or conventional loading) have not influenced the survival or success rate. Conventional loading of implants placed in healed sites was the most documented study protocol and remains the standard of care. The results indicated that minor dimensional changes in the posterior single tooth gap were inevitable during the three months following implant placement. Minor antagonist tooth extrusion happened in all the cases, while the mesial-distal distance of the tooth-missing site showed a tendency to decrease. These results partially confirmed the outcomes of some previous studies (Garcia-Herraiz et al., 2017; Gragg et al., 2001). With a single tooth loss, the remaining teeth especially the neighboring and antagonist teeth had a tendency to rearrange to establish a new equilibrium state, which might lead to the reduction of the tooth-missing gap (Garcia-Herraiz et al., 2012). Even after implant restoration, the dynamic position change of neighboring and antagonist teeth could cause implant infraposition (IIP) and proximal contact point (PCP) loss (Papageorgiou, Eliades, \& Hammerle, 2018), and should be monitored constantly at patients' follow-up.

In the present study, the mean mesial-distal distance reduction of the tooth-missing site $(37.62 \mu \mathrm{m})$ three months following implant placement was significantly smaller than that observed 
three months following natural tooth extraction $(672.30 \mu \mathrm{m})($ Garcia-Herraiz et al., 2017). The difference could be explained by different time points chosen for the baseline. In this study, the baseline of measurement was set at equal to or more than three months after tooth extraction, and in most of the participants, the posterior teeth have already been missing for several years. Therefore, the period of significant dimension change due to active bone remodeling following tooth extraction was avoided in this study (Garcia-Herraiz et al., 2017; Gragg et al., 2001).

To our knowledge, the present study is the first of its kind using intraosseous implants as reference and a digital method to quantitively analyze the movement of neighboring teeth. The implant provided a fixed reference point to define reproducible reference planes for precise measurements. Three-dimensional registration technology was used to duplicate the reference plane in the same position in both the baseline and follow-up digital models to further improve measurement accuracy. Thus, the migration of neighboring and antagonist teeth relative to the implant position can be analyzed separately on the scale of microns. Compared with stone models, the digital models can be stored for a long time without information loss and can be used to analyze the change of dentition for this group of patients in the future. The digital measurement protocol using engineering software program has been proved to be an acceptable form of measurement with statistical validation (Zeller, Guichet, Kontogiorgos, \& Nagy, 2019).

For the 18 mesial neighboring teeth moving distally, the average moving distance was significantly larger than that of the 16 distal neighboring teeth moving distally. The lack of support in-between the mesial and distal neighboring teeth could be the reason for this difference.

In this study, more than 75 percent of the neighboring and antagonist teeth showed less than $100 \mu \mathrm{m}$ linear tooth migration (Table 2). The mesial-distal dimensional change of the tooth-missing sites was minimal. These findings provided evidence for clinical adoption of a more time-efficient fully digital workflow in implant restoration of single posterior missing tooth (Guo et al., 2019; Pan et al., 2019). It has been shown that the single implant crown can be fabricated from digital impressions taken immediately after implant surgery by means of a model-free, laboratory-based fully digital workflow, and delivered 3 months after implant placement. The chairside time for the crown delivery was similar to that of implant crowns made from 
conventional impression taken 3 months after implant placement (Pan et al., 2019). With the data from this study, it was clarified that the fit of the implant crown was not affected by the minor migration of the neighboring and antagonist teeth during the 3 months. The clinical relevance of the findings from this study was to provide appropriate designing parameters for implant crown in fully digital workflow, and the fit of the single implant crowns can be further improved.

Participants' sexes and upper or lower jaw did not significantly affect the dimensional change of the tooth-missing site, and this was similar to the result of García Erraiz et al. (Garcia-Herraiz et al., 2017).

When participants were divided into three age subgroups, the mesial-distal dimensional change of tooth-missing site in the younger-than-30-years subgroup was significantly larger than that in the older-than-50-years group $(P=0.023)$. The present results are in accordance with a previous study showing a more pronounced bone remodeling in a young population resulting in larger mesial-distal tooth-missing gap reduction (Verna, 2016).

It should be noted that this study is a single-group prospective cohort study based on a previous randomized controlled clinical trial (RCT), therefore it is a spin-off use of data from a RCT (Pan et al., 2019). Both the registration and the randomization aspect of this study pertain to the primary research question. Limitations of the present study include that intraoral scan, registration process and definition of reference lines on different digital model could introduce errors in the measurement, and these errors were not assessed in this study. Due to the definitions of the reference lines and the central axis of the scan body, the distance values were automatically displayed by the digital analysis software instead of manual measurement, the repeated measurement was not performed. This study only evaluated the linear change between fixed reference lines/points on neighboring and antagonist teeth, without indicating the rotational and inclination movement. In addition, the influence of the type of interocclusal relationship was not tested because of the low number of patients.

\section{Conclusion}

The dimensions of posterior tooth-missing sites decreased over an observation period of three months. 


\section{Acknowledgments}

The authors express their gratitude to Dai Tong (Clinical professor, Department of Prosthodontics, Peking University School and Hospital of Stomatology), Bing Wang, Pengfei

Wang, and Wenhui Gao (Dental Technicians, Laboratory Center, Peking University School and Hospital of Stomatology) for the acquisition of the digital model.

\section{Conflict of interest}

The authors declare no conflict of interest.

This article is protected by copyright. All rights reserved 


\section{References}

Christou, P., \& Kiliaridis, S. (2007). Three-dimensional changes in the position of unopposed molars in adults. Eur J Orthod, 29(6), 543-549. http://doi.org/10.1093/ejo/cjm036

Craddock, H. L., Youngson, C. C., Manogue, M., \& Blance, A. (2007). Occlusal changes following posterior tooth loss in adults. Part 2. Clinical parameters associated with movement of teeth adjacent to the site of posterior tooth loss. J Prosthodont, 16(6), 495-501. http://doi.org/10.1111/j.1532-849X.2007.00223.x

Gallucci, G. O., Hamilton, A., Zhou, W., Buser, D., \& Chen, S. (2018). Implant placement and loading protocols in partially edentulous patients: A systematic review. Clin Oral Implants Res, 29 Supp/ 16, 106-134. http://doi.org/10.1111/clr.13276

Garcia-Herraiz, A., Leiva-Garcia, R., Canigral-Ortiz, A., Silvestre, F. J., \& Garcia-Anton, J. (2012). Confocal laser scanning microscopy for the study of the morphological changes of the postextraction sites. Microsc Res Tech, 75(4), 513-519. http://doi.org/10.1002/jemt.21085

Garcia-Herraiz, A., Silvestre, F. J., Leiva-Garcia, R., Crespo-Abril, F., \& Garcia-Anton, J. (2017). Post-extraction mesio-distal gap reduction assessment by confocal laser scanning microscopy - a clinical 3-month follow-up study. J Clin Periodontol, 44(5), 548-555. http://doi.org/10.1111/jcpe.12706

Gragg, K. L., Shugars, D. A., Bader, J. D., Elter, J. R., \& White, B. A. (2001). Movement of teeth adjacent to posterior bounded edentulous spaces. Journal of Dental Research, 80(11), 2021-2024. http://doi.org/10.1177/00220345010800111401

This article is protected by copyright. All rights reserved 
Guo, D. N., Liu, Y. S., Pan, S. X., Wang, P. F., Wang, B., Liu, J. Z., . . Zhou, Y. S. (2019). Clinical Efficiency and Patient Preference of Immediate Digital Impression after Implant Placement for Single Implant-Supported Crown. Chin J Dent Res, 22(1), 21-28. http://doi.org/10.3290/j.cjdr.a41771

Joda, T., Ferrari, M., \& Bragger, U. (2017). Monolithic implant-supported lithium disilicate (LS2) crowns in a complete digital workflow: A prospective clinical trial with a 2-year follow-up. Clin Implant Dent Relat Res, 19(3), 505-511. http://doi.org/10.1111/cid.12472

Kiliaridis, S., Lyka, I., Friede, H., Carlsson, G. E., \& Ahlqwist, M. (2000). Vertical position, rotation, and tipping of molars without antagonists. International Journal of Prosthodontics, 13(6), 480-486. http://doi.org/10.1046/j.1365-2591.2000.00365.x

Lindskog-Stokland, B., Hansen, K., Tomasi, C., Hakeberg, M., \& Wennstrom, J. L. (2012). Changes in molar position associated with missing opposed and/or adjacent tooth: a 12-year study in women. $J$ Oral Rehabil, 39(2), 136-143. http://doi.org/10.1111/j.1365-2842.2011.02252.x

Love, W. D., \& Adams, R. L. (1971). Tooth Movement into Edentulous Areas. Journal of Prosthetic Dentistry, 25(3), 271-\&. http://doi.org/10.1016/0022-3913(71)90188-0

Lyka, I., Carlsson, G. E., Wedel, A., \& Kiliaridis, S. (2001). Dentists' perception of risks for molars without antagonists. Swedish Dental Journal, 25(2), 67-73.

Moreira, A. H., Rodrigues, N. F., Pinho, A. C., Fonseca, J. C., \& Vilaca, J. L. (2015). Accuracy Comparison of Implant Impression Techniques: A Systematic Review. Clin Implant Dent Relat Res, 17 Supp/ 2(S2), e751-764. http://doi.org/10.1111/cid.12310

Muhlemann, S., Kraus, R. D., Hammerle, C. H. F., \& Thoma, D. S. (2018). Is the use of digital technologies

This article is protected by copyright. All rights reserved 
for the fabrication of implant-supported reconstructions more efficient and/or more effective than conventional techniques: A systematic review. Clin Oral Implants Res, 29 Suppl 18, 184-195. http://doi.org/10.1111/clr.13300

Pan, S., Guo, D., Zhou, Y., Jung, R. E., Hammerle, C. H. F., \& Muhlemann, S. (2019). Time efficiency and quality of outcomes in a model-free digital workflow using digital impression immediately after implant placement: A double-blind self-controlled clinical trial. Clin Oral Implants Res, 30(7), 617-626. http://doi.org/10.1111/clr.13447

Papageorgiou, S. N., Eliades, T., \& Hammerle, C. H. F. (2018). Frequency of infraposition and missing contact points in implant-supported restorations within natural dentitions over time: A systematic review with meta-analysis. Clin Oral Implants Res, 29 Suppl 18, 309-325. http://doi.org/10.1111/clr.13291

Peters, M. C., Delong, R., Pintado, M. R., Pallesen, U., Qvist, V., \& Douglas, W. H. (1999). Comparison of two measurement techniques for clinical wear. $J$ Dent, 27(7), 479-485. http://doi.org/10.1016/s0300-5712(99)00027-5

Shah, S., Sundaram, G., Bartlett, D., \& Sherriff, M. (2004). The use of a 3D laser scanner using superimpositional software to assess the accuracy of impression techniques. J Dent, 32(8), 653-658. http://doi.org/10.1016/j.jdent.2004.07.005

Shugars, D. A., Bader, J. D., Phillips, S. W., Jr., White, B. A., \& Brantley, C. F. (2000). The consequences of not replacing a missing posterior tooth. Journal of the American Dental Association, 131(9), 1317-1323. http://doi.org/10.14219/jada.archive.2000.0385

Verna, C. (2016). Regional Acceleratory Phenomenon. Front Oral Biol, 18, 28-35. 
http://doi.org/10.1159/000351897

Weinstei.S. (1967). Minimal Forces in Tooth Movement. American Journal of Orthodontics, 53(12), 881-\&. http://doi.org/10.1016/0002-9416(67)90163-7

Zeller, S., Guichet, D., Kontogiorgos, E., \& Nagy, W. W. (2019). Accuracy of three digital workflows for implant abutment and crown fabrication using a digital measuring technique. Journal of Prosthetic Dentistry, 121(2), 276-284. http://doi.org/10.1016/j.prosdent.2018.04.026

This article is protected by copyright. All rights reserved 


\section{Tables}

Table 1 Tooth-missing position and number of teeth in each position

\begin{tabular}{|l|l|l|l|l|l|l|l|l|l|l|}
\hline Tooth-missing position & 14 & 15 & 16 & 24 & 26 & 35 & 36 & 45 & 46 & Total \\
\hline Number of teeth & 1 & 2 & 7 & 1 & 4 & 2 & 13 & 1 & 9 & 40 \\
\hline
\end{tabular}

This article is protected by copyright. All rights reserved 
Table 2 Moving distance of mesial and distal neighboring teeth (absolute value, $\mu \mathrm{m}$ )

\begin{tabular}{|c|c|c|c|c|c|c|}
\hline \multirow{2}{*}{ Neighboring teeth } & \multicolumn{3}{|c|}{ Moving mesially } & \multicolumn{3}{|c|}{ Moving distally } \\
\cline { 2 - 3 } & $\mathrm{n}$ & Median $\left(\mathrm{Q}_{25} / \mathrm{Q}_{75}\right)(\mu \mathrm{m})$ & $P$ & $\mathrm{n}$ & Median $\left(\mathrm{Q}_{25} / \mathrm{Q}_{75}\right)(\mu \mathrm{m})$ & $P$ \\
\hline Mesial & 22 & $41.92(19.58 / 69.98)$ & \multirow{2}{*}{0.071} & 18 & $85.17(51.87 / 99.20)$ & \multirow{2}{*}{$0.003 *$} \\
\hline Distal & 24 & $58.74(35.21 / 99.28)$ & & 16 & $49.95(17.33 / 64.65)$ & \\
\hline
\end{tabular}

$* P<0.05$ 
Table 3 Mesial-distal and occlusal-gingival dimensional change of the tooth-missing site among the three age subgroups (the negative value represents a distance reduction)

\begin{tabular}{|c|c|c|c|c|c|}
\hline \multicolumn{2}{|c|}{} & \multicolumn{2}{c|}{ Mesial-distal dimensional change } & \multicolumn{2}{c|}{ Occlusal-gingival dimensional change } \\
\cline { 2 - 7 } & $\mathrm{n}$ & Median $\left(\mathrm{Q}_{25} / \mathrm{Q}_{75}\right)(\mu \mathrm{m})$ & $\mathrm{n}$ & Median $\left(\mathrm{Q}_{25} / \mathrm{Q}_{75}\right)(\mu \mathrm{m})$ \\
\hline \multirow{2}{*}{ Age } & $<30$ & 8 & $-102.42(-218.78,-54.04)$ & 8 & $-63.5(-95.50,-40.50)$ \\
\cline { 2 - 7 } subgroups & $30-50$ & 18 & $-13.68(-73.41,41.96)$ & 18 & $-51.50(-92.75,-33.00)$ \\
\cline { 2 - 7 } (years) & $>50$ & 14 & $-6.35(-36.73,57.25)$ & 14 & $-62.5(-81.50,-52.50)$ \\
\hline \multicolumn{2}{|c|}{$P$} & & $0.03 *$ & & 0.74 \\
\hline
\end{tabular}

$* P<0.05$

This article is protected by copyright. All rights reserved 
Table 4 Mesial-distal and occlusal-gingival dimensional change of the single posterior tooth-missing site according to sex, tooth-missing position, and jaw position.

\begin{tabular}{|c|c|c|c|c|c|c|c|}
\hline \multicolumn{2}{|c|}{ Factors } & \multicolumn{3}{|c|}{ Mesial-distal dimensional change } & \multicolumn{3}{|c|}{ Occlusal-gingival dimensional change } \\
\hline & & $\mathrm{n}$ & $\operatorname{Median}\left(\mathrm{Q}_{25} / \mathrm{Q}_{75}\right)(\mu \mathrm{m})$ & $P$ & $\mathrm{n}$ & $\operatorname{Median}\left(\mathrm{Q}_{25} / \mathrm{Q}_{75}\right)(\mu \mathrm{m})$ & $P$ \\
\hline \multirow[t]{2}{*}{ Sex } & Male & 19 & $-20.86(-38.60,39.97)$ & \multirow[t]{2}{*}{0.27} & 19 & $-61.00(-79.00,-36.00)$ & \multirow[t]{2}{*}{0.45} \\
\hline & Female & 21 & $-41.84(-129.58,32.58)$ & & 21 & $-66.00(-110.50,-37.00)$ & \\
\hline \multirow{2}{*}{$\begin{array}{c}\text { Tooth-missing } \\
\text { position }\end{array}$} & Premolar & 7 & $-38.60(-129.93,8.55)$ & \multirow[t]{2}{*}{0.58} & 7 & $-41.00(-62.00,-27.00)$ & \multirow[t]{2}{*}{0.05} \\
\hline & Molar & 33 & $-21.25(-69.70,44.05)$ & & 33 & $-63.00(-93.50,-41.50)$ & \\
\hline \multirow[t]{2}{*}{ Jaw position } & Upper & 15 & $-20.87(-63.00,35.97)$ & \multirow[t]{2}{*}{0.98} & 15 & $-63.00(-79.00,-41.00)$ & \multirow[t]{2}{*}{0.80} \\
\hline & Lower & 25 & $-36.57(-88.94,50.01)$ & & 25 & $-58.00(-95.50,-35.50)$ & \\
\hline
\end{tabular}




\section{Figure Legends}

Figure 1. IOS digital model acquisition. a, Intraoral scan made immediately after implant placement. b, Intraoral scan made 3 months following implant placement. c, Digital baseline model (BM). d, Digital follow-up model (FM)

Figure 2. Measurement of neighboring teeth movement. a, Parallel buccal plane and reference plane (RP) generated with RP running through central axis of scan body. $b$, Reference lines in the RP parallel to central axis of scan body and tangential to mesial and distal neighboring teeth. c, Distances between central axis of scan body and reference lines measured mesially (D1) and distally (D2).

Figure 3. Measurement of antagonist teeth extrusion. A, Five featuring points on antagonist tooth. b, Superimposition of BM and FM using best fit alignment of neighboring teeth. c, Vector change of reference points between BM (purple line) and FM (black line) were measured.

Figure 4. Mesial-distal reduction of tooth-missing site was significantly larger in patients younger than 30 years compared to those older than 50 years. No significant difference was found in occlusal gingival dimensional change of the tooth-missing site among three age subgroups 


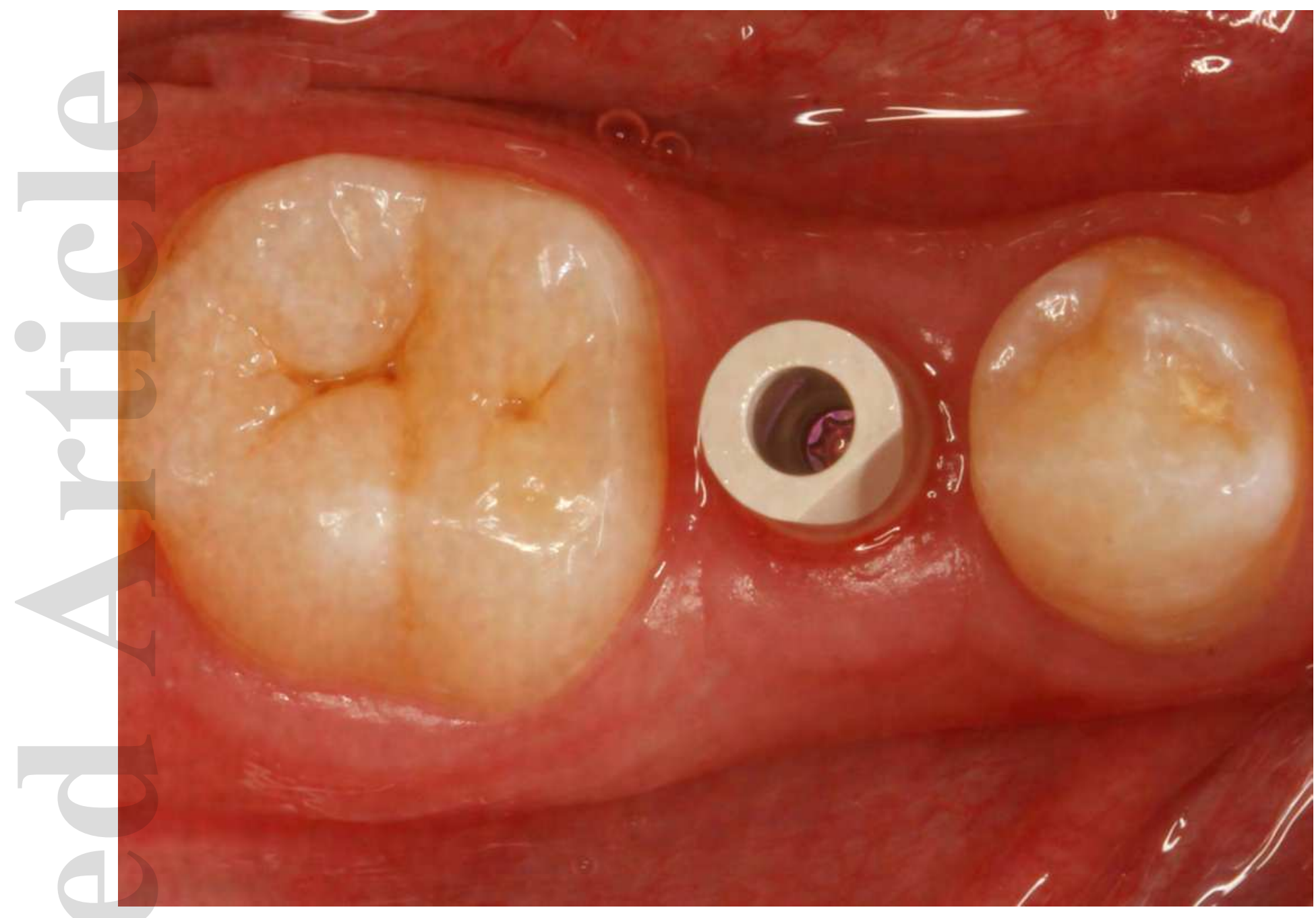

clr_13694_f1b.tif

This article is protected by copyright. All rights reserved 


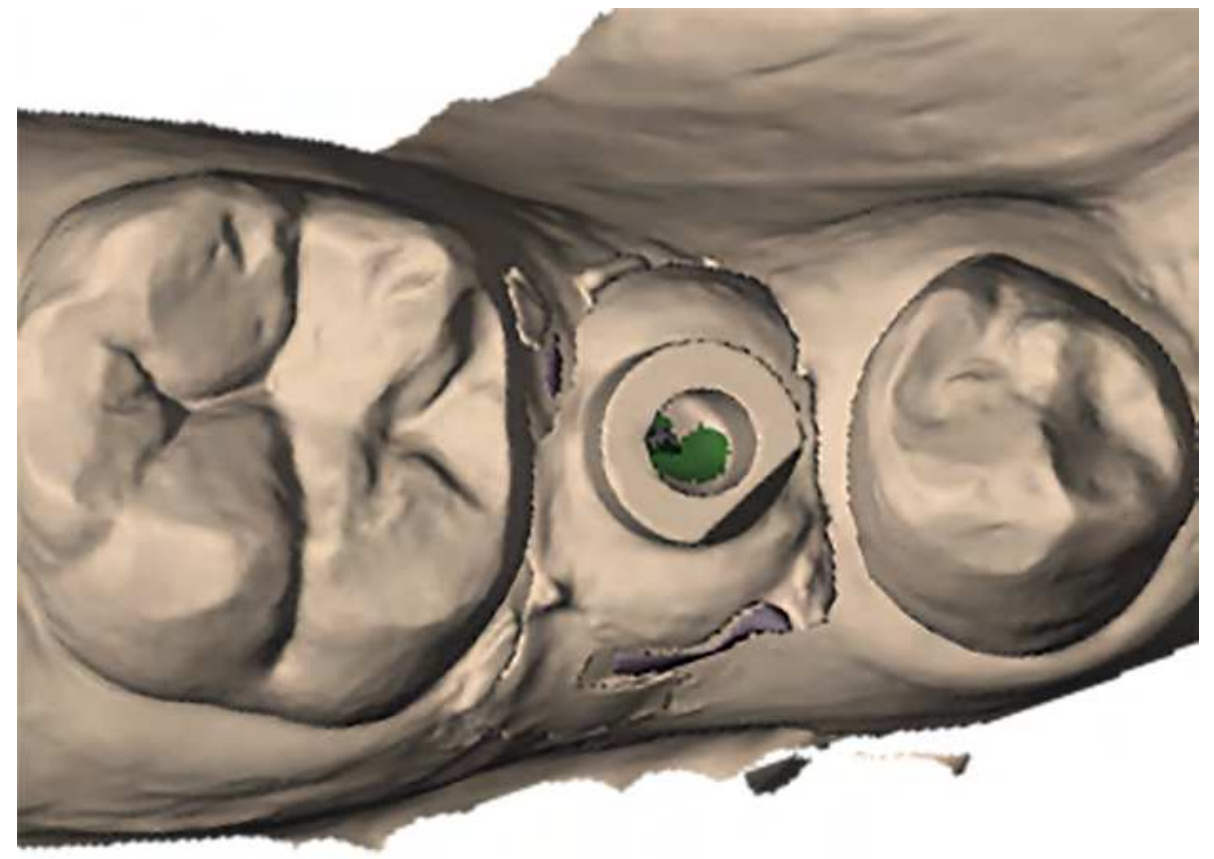

clr_13694_f1c.tif 


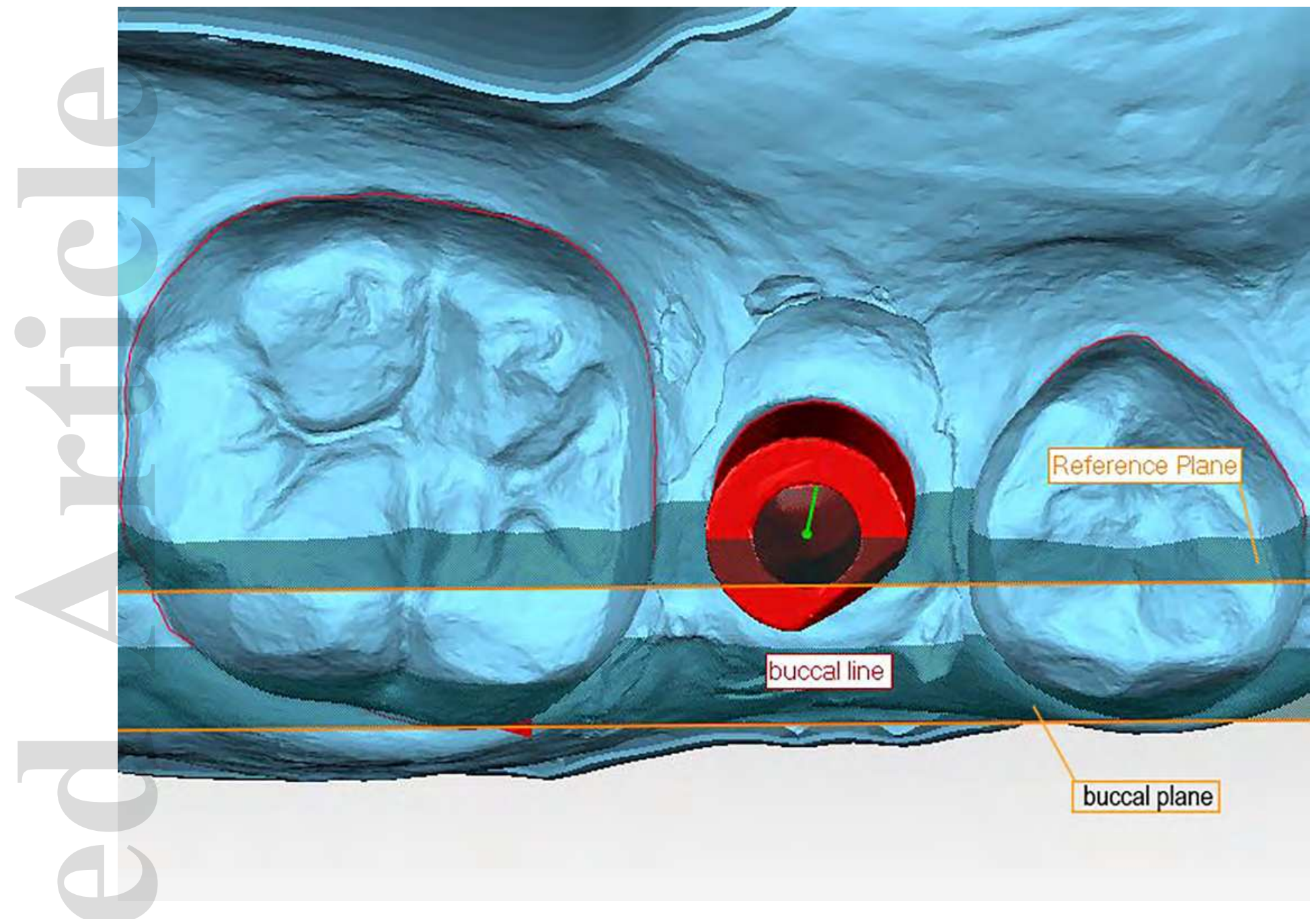

clr_13694_f2a.tif

This article is protected by copyright. All rights reserved 

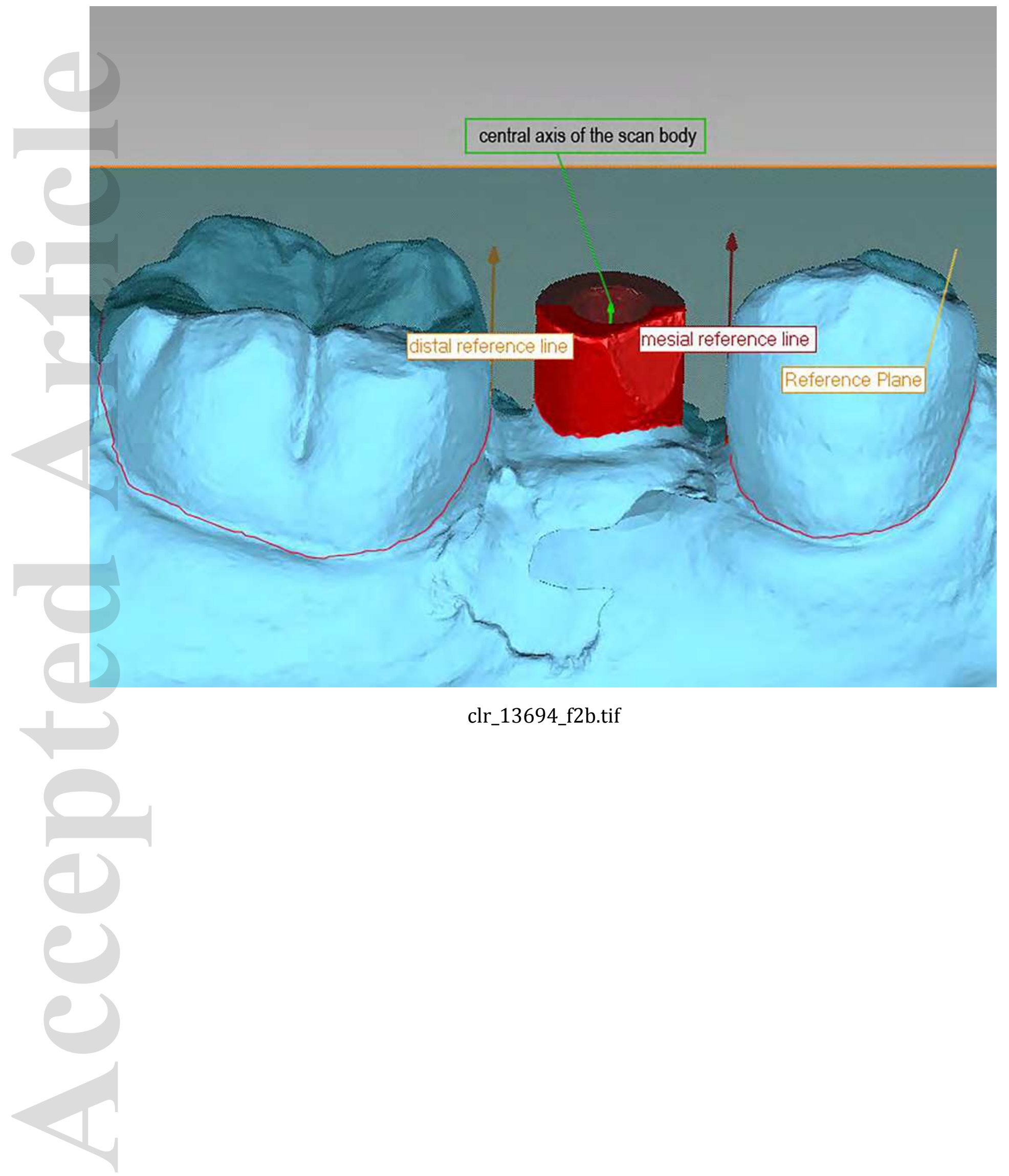

This article is protected by copyright. All rights reserved 


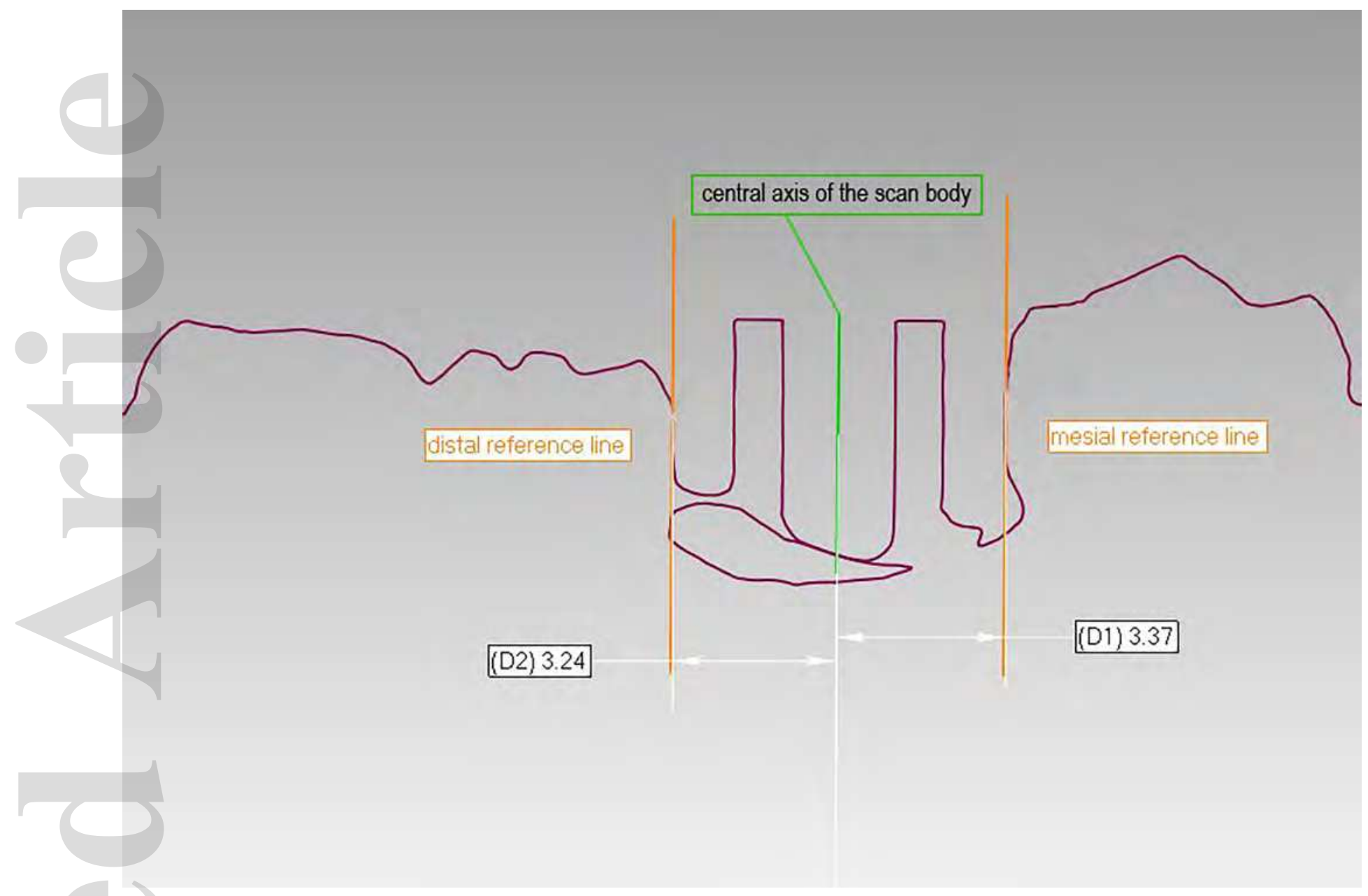

clr_13694_f2c.tif

This article is protected by copyright. All rights reserved 


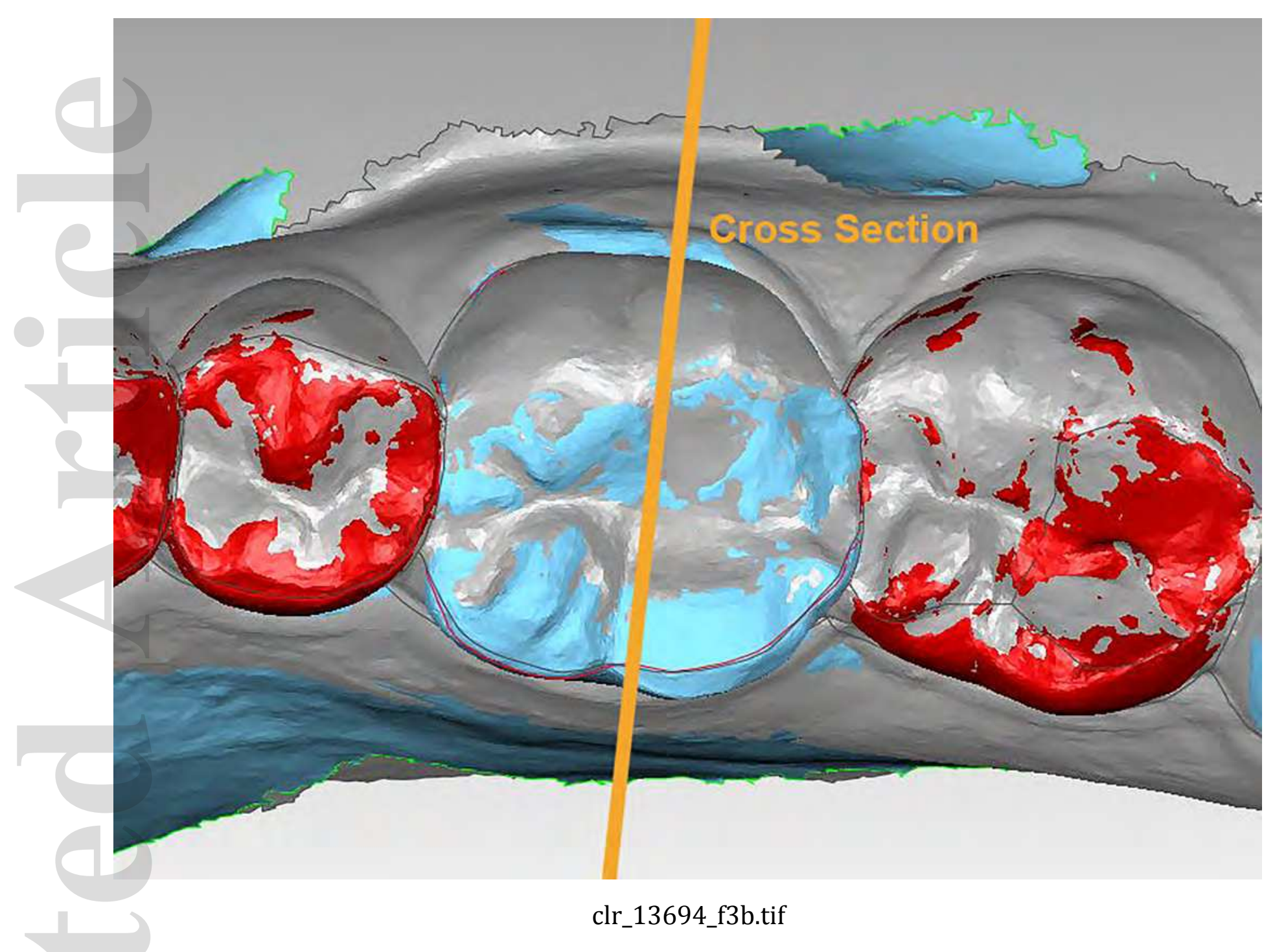

This article is protected by copyright. All rights reserved 


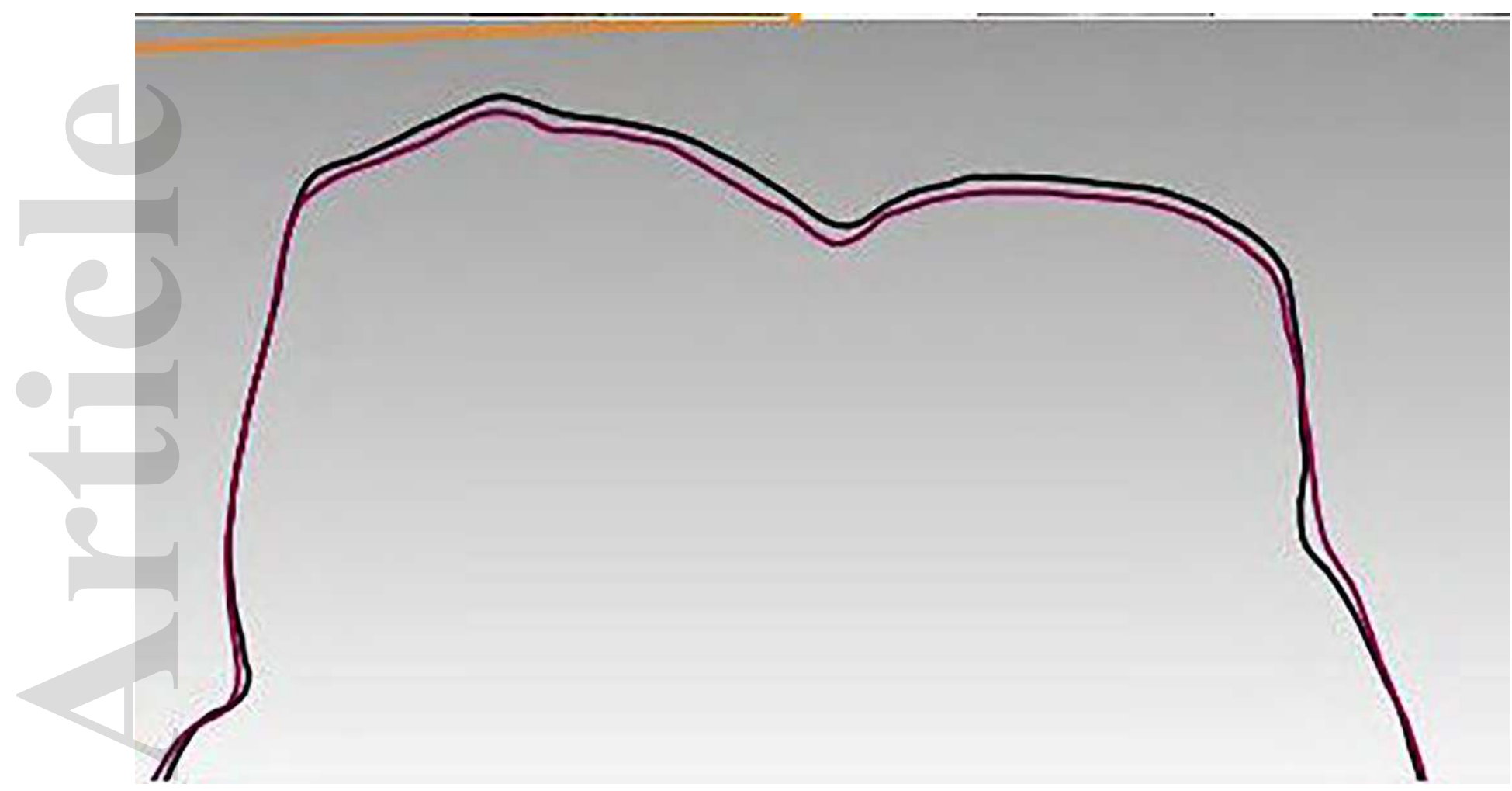

clr_13694_f3c.tif

This article is protected by copyright. All rights reserved 


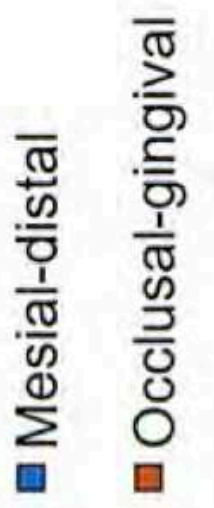

요

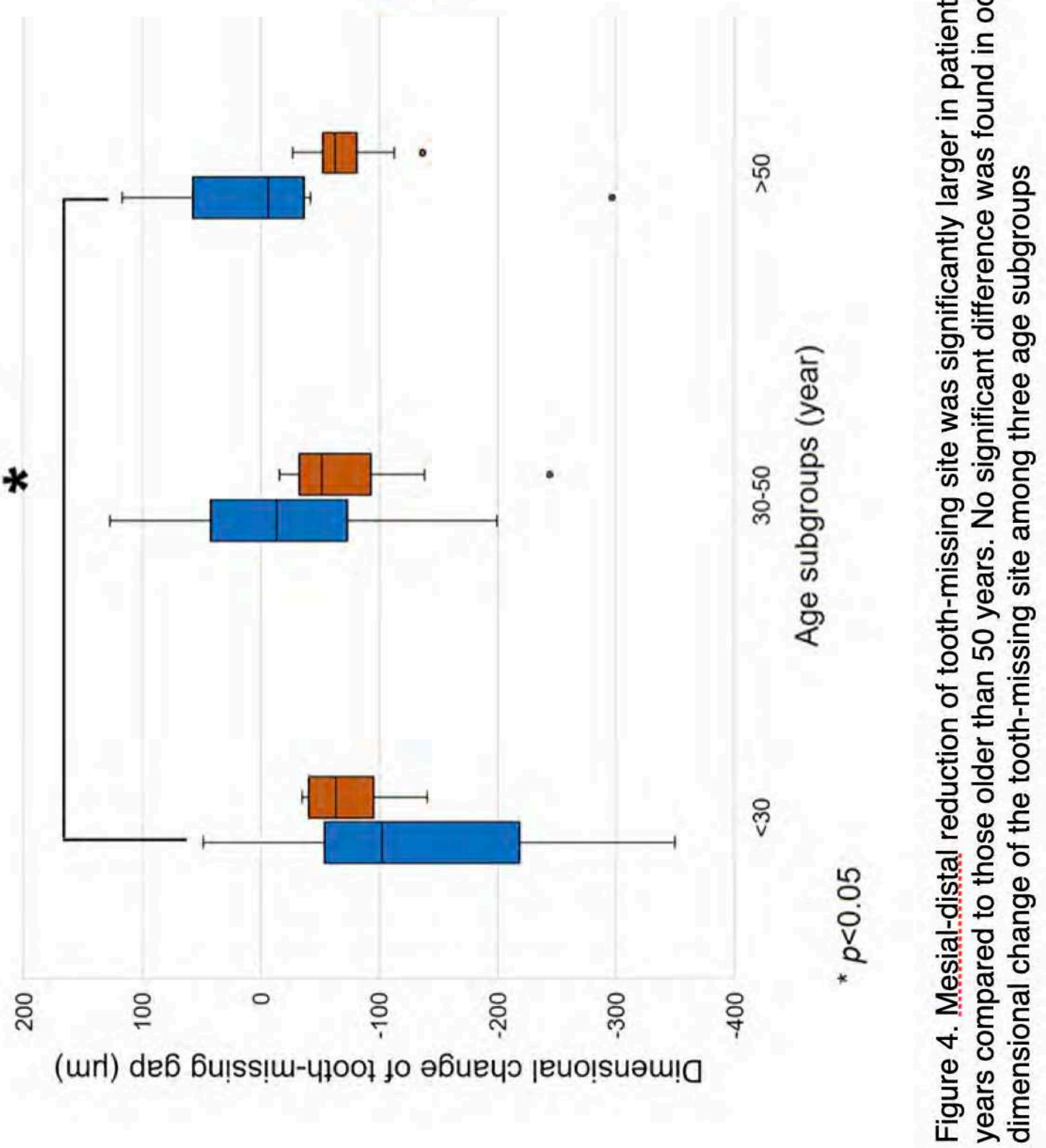

